\title{
Kernos
}

Revue internationale et pluridisciplinaire de religion grecque antique

$17 \mid 2004$

Varia

\section{Les origines cycladiques de la geranos : sur un pendentif en pierre du Délion de Paros}

\section{Zozie Papadopoulou}

\section{(2) OpenEdition \\ Journals}

\section{Édition électronique}

URL : http://journals.openedition.org/kernos/1408

DOI : 10.4000/kernos. 1408

ISSN : 2034-7871

\section{Éditeur}

Centre international d'étude de la religion grecque antique

\section{Édition imprimée}

Date de publication : 1 janvier 2004

ISSN : 0776-3824

\section{Référence électronique}

Zozie Papadopoulou, «Les origines cycladiques de la geranos : sur un pendentif en pierre du Délion de Paros », Kernos [En ligne], 17 | 2004, mis en ligne le 22 avril 2011, consulté le 01 mai 2019. URL : http:// journals.openedition.org/kernos/1408; DOI : 10.4000/kernos.1408 


\section{Les origines cycladiques de la geranos: sur un pendentif en pierre du Délion de Paros}

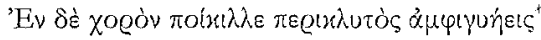

En 1960, pendant les travaux de clôture et d'aménagement du Délion de Paros, quand, "enfin », comme l'écrit de manière significative N. Zapheiropoulos, s'est présentée l'opportunité de son acquisition, on a découvert, parmi une quantité inattendue de trouvailles, un pendentif en forme de plaque, en pierre semi-transparente verdâtre, identifiée comme de la stéatite ${ }^{1}$, avec une décoration gravée sur tous les côtés².

Le pendentif a une forme trapézoïdale. L'un de ses coins supérieurs est cassé, alors qu'une petite partie du coin inférieur du revers est abîmée. De fortes traces d'usure apparaissent sur l'une des faces latérales où une partie de la décoration n'est pas conservée. L'usure est due à l'utilisation de l'objet. Sur' la face supérieure, des fentes hémisphériques définissent la partie à attacher sur laquelle ont été percés, à des hauteurs différentes, deux petits trous qui transpercent le tout $(0,0001 \mathrm{~m}$ et $0,0002 \mathrm{~m})$. Dimensions : ht 0,0395 m, épaisseur 0,004-0,0045 m, taille plaque $0,032 \mathrm{~m}$. La décoration du pendentif se développe sur les six faces.

Sur la face A sont représentés deux personnages nus dansant vers la droite. La tête et les membres sont représentés de profil, le torse triangulaire, de face. Le nez et le menton sont fortement saillants, surtout sur la figure

* HoM., Il. XVII, 590. Cet article a été publié en grec dans les Mélanges Cbr: Doumas (éd. par A. Vlachopollos, K. BirTaChA), Athènes, 2003. Je veux remercier chaleureusement Dr. Ph. Zapheiropoulou, éphore honoraire des Antiquités des Cyclades, qui m'a donné l'autorisation de publier le pendentif et des informations précieuses sur les recherches de $\mathrm{N}$. Zapheiropoulos au Délion de paros. Je lui dois encore davantage pour les années passées à travailler avec elle dans les Cyclades et tout ce que j'ai vu et appris là-bas. Tout aussi inestimable est l'aicle de Prof. L. Marangou, ainsi que celle de Prof. N. Kourou, qui a eu la patience de lire le texte et de me faire des suggestions particulièrement utiles; toute faiblesse dans le traitement du sujet m'incombe bien évidemment. Les dessins précis de toutes les faces du pendentif sont dus à la conservatrice des antiquités E. Papathoma (p. 174) et la qualité des photographies à E. Heliadis (p. 156-157). Cette étude n'aurait pu être menée à bien sans l'obtention de la bourse S.J. Seeger du programme des études helléniques de l'Université de Princeton, que je remercie également chaleureusement.

1 Pour le type de pierre, voir BOARDMAN (1970), p. 118.

2 D'après le catalogue du musée de Paros ( $n^{\circ}$ inv. 1590), la petite plaque a été découverte le 13 juin 1960 dans l'enclos sud du Délion. N. Zapheiropoutos (1962), p. 246, précise que cette plaquette, comme les autres trouvailles, ont été mises au jour «soit pendant la destruction des murs d'enceinte [plus récents, à l'intérieur du site archéologique], soit au cours du creusement du terrain pour la consolidation des bâtiments du sanctuaire ou l'établissement de la zone de fouille." " 
droite, alors que la tête de la figure gauche penche légèrement vers l'arrière. Les bras se lèvent au-dessus de la tête en formant un cercle. On ne peut pas distinguer d'articulation au coude. Les mains prennent la forme d'une fourche et se trouvent très près l'une de l'autre, donnant l'impression qu'elles vont se joindre. Les jambes sont représentées écartées, les genoux fléchis, la jambe gauche se pose sur le sol de manière stable, alors que la droite est encore en l'ail:

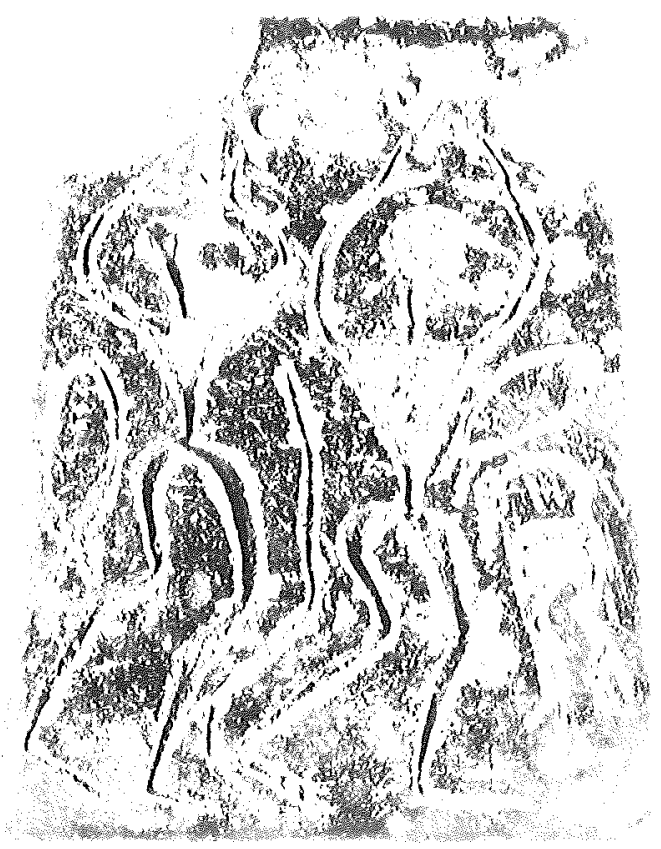

Quoique les deux personnages soient représentés d'une manière comparable, certaines petites différences se marquent : la figure de droite a le torse et la tête plus grands et le cou plus haut; au contraire, la tête et le torse du danseur de gauche sont plus petits, mais les jambes sont tellement hautes qu'il paraît avoir une taille équivalente à celle de la figure de droite ${ }^{3}$. Entre les deux danseurs apparaît un dessin linéaire qui, dans sa partic inférieure, se présente comme un zigzag, suivant le dessin des jambes des deux personnages, alors que, vers le haut, il est presque rectiligne. Au fond, de part et d'autre des deux figures se distinguent, à droite et à gauche, des poissons qui nagent vers le haut et vers la droite. En-dessous du poisson de droite se trouve un oiseau aquatique haut sur pattes, ces pattes se terminant par une fourche, et avec un long cou tourné vers l'arrière, vers les danseurs.

La plus grande partie de la face B est occupée par un labyrinthe circulaire du type dit «cnossien», composé de sept couloirs. Au-dessus du labyrinthe, une décoration en forme de goutte représente un tracé hélicoïdal rappelant le mouvement boustrophédon des inscriptions. Les trous d'attache du pendentif ont été percés sur deux des lignes de ce tracé. Dès lors, ils sont placés d'une manière asymétrique qui semble volontaire. Le long du côté gauche de la face $\mathrm{B}$ a été gravée une ondulation. Une ligne comparable apparaît également en-

3 Une différenciation comparable se remarque également pour la dernière figure (à gauche pour le spectateur) dans un groupe de danseurs représentés sur une pyxis laconienne du sanctuaire d'Apollon Amyklaios (Musée archéologique d'Athènes, 234) : la figure est représentée avec un petit torse, des jambes plus courtes, mais un cou si long que sa hauteur est identique à celle des autres (fin vil" s. av. J.-C.). Crowhurst (1963), p. 81 sq., nº 69; TÖlle (1964), p. 49; COLDSTREAM (1968a), p. 217-218, pl. 46n. 
dessous du labyrinthe circulaire, ligne qui se divise à l'une de ses extrémités. La même chose se passe à l'une des extrémités du tracé hélicoïdal, juste audessus du labyrinthe.

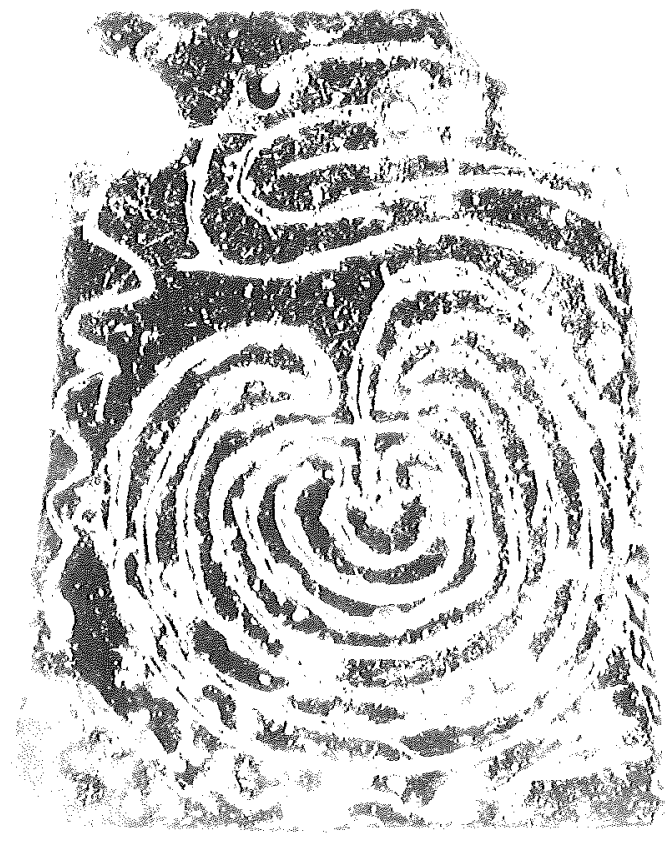

Des ondulations comparables à celles de la face $B$ (avec des extrémités simples) apparaissent également sur le côté inférieur et sur les deux longs côtés du pendentif. Enfin, le côté étroit supérieur porte une ligne gravée en zigzag.

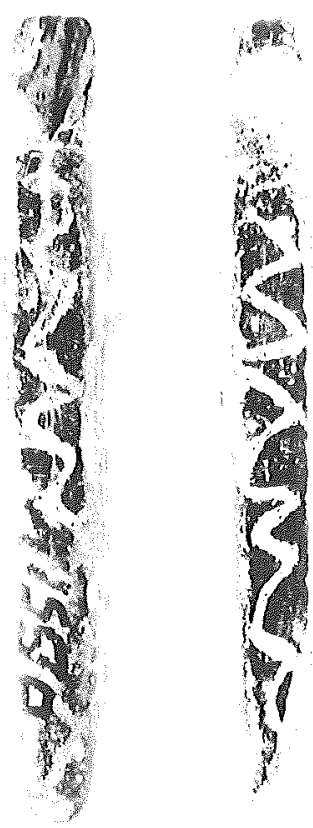

\section{Analyse iconographique et technique}

\subsection{Les figures dansantes}

Dans l'art cycladique du géométrique récent, les scènes de danse connues jusqu'ici se limitent à des représentations de danses de femmes en groupe sur des vases d'ateliers naxiens ${ }^{4}$. Sur le pendentif de Paros, les deux personnages

4 Amphore de Naxos avec représentation de danse de l'Artémision de Délos (Musée de Délos, B. 4208, groupe Bc; Délos XV, n 6, p. 87, fig. 43, 56). Pour un deuxième exemple de représentation chorale du même groupe, voir Lambrinoudakis (1983), p. 167, fig. 21. 
qui dansent sont très probablement masculins : ils sont représentés nus, et même si cet élément ne constitue pas un facteur décisif pour l'identification, l'absence de tout détail anatomique ou autre (vêtement, coiffure), aussi bien que le genre de mouvement qui est effectué, plaident en faveur d'une identification masculine $e^{5}$. La manière de représenter les traits du visage, mais aussi l'inclinaison légère de la tête vers l'arrière chez le personnage de gauche", créent l'impression que l'artisan a voulu figurer la bouche ouverte et donc que les deux formes sont très probablement représentées en train de chanter ${ }^{7}$ (ou, plus précisément, de produire de manière rythmique quelques sons, étant donné que la chanson pourrait avoir une signification très différente de celle d'aujourd'huil ${ }^{8}$. De même, la manière de représenter les jambes, avec les genoux fléchis, avec un pied en l'air, aussi bien que celle des mains, très proches l'une de l'autre, prêtes à se joindre, rendent plus forte la sensation rythmique de la danse.

Les deux figures présentent des ressemblances avec des types iconographiques d'Attique et d'Eubée essentiellement'. La représentation la plus ancienne de l'époque géométrique, présentant un mouvement de danse analogue (notamment au niveau des jambes), se trouve sur le canthare $727 \mathrm{du}$ Musée National de Copenhague ${ }^{10}$ : on y voit quatre figures masculines, à gauche, le musicien à la lyre et, à droite, trois danseurs. Du premier, qui effectue un mouvement de soliste par un grand saut ${ }^{11}$, ne subsistent que les jambes. Les deux autres danseurs "applaudissent" (pas au-dessus de la tête, mais à hauteur du sternum), alors que le mouvement, en regard du reste, est équivalent à ceux des danseurs du pendentif de Paros ${ }^{12}$. De même, dans une

5 Pour la détermination du sexe, voir BENSON (1970), p. 106; AH.BERG (1971), p. $72 s q$. Concernant plus particulièrement les danseurs isolés ( «solo») dans les représentations du géométrique récent, voir CrowhuRst (1963), p. 21 et $22, \mathrm{n} .2$, qui remarque que ce sont toujours des hommes.

" Pour les relations des cette position de la tête avec la chanson, chez les idoles protocycladiques de musiciens, voir Marangou (1999), p. 25.

Cf: la $3^{\circ}, 5^{\circ}, 7^{*}, 12^{*}$ (de la gauche) figure masculine dans la zone inférieure du col de l'hydrie proto-attique CVA Berlin 1, pl. 1, fig. 1. Crowhurst (1963), p. 33, no 37 et fig. 4. De mềme, les musiciens des groupes choraux sur le canthare NM 727 de Copenhague : la représentation du nez et du menton du musicien qui accompagne le chœur féminin suggère peut-être une chanson (au contraire, chez les musiciens du chour masculin, seul le menton est représenté) : CROWHURsT (1963), p. 23. Une représentation équivalente des traits du visage du citharode sur le tesson d'Argos C 3943 : Tölle (1964), p. 43; AhlBerg (1987), p. 64-67, fig. 17. Contra Crowhurst (1963), p. 70. Pour des exemples de musiciens et de danseurs représentés en train de chanter sur des vases géométriques, $c f$. aussi WhGNer (1968), pl. UIIb (le musicien), pl. UIIIb (le dernier danseur), pl. UVb (les danseurs), etc.

${ }^{8}$ Pour une approche interculturelle du terme "chanson ", voir NAGY (1990), chap. 1.

9 SChweitzer (1971), p. 56; Coldstream (2003), p. 122, 124-125.

19) WegNer (1949), p. 222, fig. 1a-b; DAVISON (1961), p. 83 sq., pl. 128; CrOWHURST (1963), p. 22; TÖllE (1964), p. 14; AHLBERG (1987), p. 55 sq.

11 AHLBERG (1987), p. 63-67, le qualifie d'acrobatique.

$12 \mathrm{C} /$. le tesson C 3943 d'Argos où les figures ont les jambes fléchies et paraissent joindre les mains à la hauteur du visage. Crowhurst (1963), p. 71, considère que, probablenent, ils bougent 
représentation qui se répète sur des diadèmes athéniens en or du troisième quart du vII e siècle av. J.-C., parmi des figures qui dansent en ligne - probablement des femmes -, des rameaux à la main, apparait un danseur. Il est de plus petite dimension, apparemment plus jeune, et il saute en l'air avec les jambes fléchies et les bras levés ${ }^{13}$. Le mouvement de la pointe des pieds est assez commun dans l'iconographie du géométrique récent (Crowhurst 1963, 215). Les sauts et les «applaudissements » caractérisent surtout les hommes sans exclure leur accomplissement aussi par des femmes (seulement pour les " applaudissements »), mais il s'agit d'un cas beaucoup plus rare. Il caractérise seulement celles qui ont une place distincte dans le groupe, à savoir la première ou la dernière du chour ${ }^{15}$. Il est possible que quelques-uns de ces mouvements fassent écho à des descriptions homériques des "tours" des

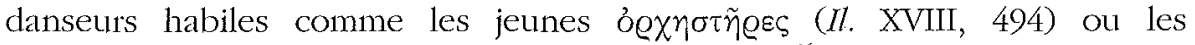

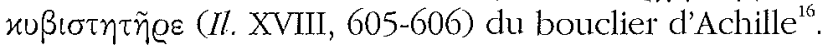

Un mouvement comparable à celui des figures du pendentif, particulièrement pour les bras, se retrouve chez les danseurs du canthare 14447 (Musée National d'Athènes) provenant d'Anavyssos : quatre danseurs se déplacent vers la droite derrière un joueur de pborminx.17. Les deux premiers et le dernier font des pas équivalents à ceux des figures du pendentif et sont prêts à « applaudir » à hauteur du visage. L'avant-dernier danseur, qui paraît compléter le mouvement de la figure abîmée du canthare 727 , saute vers le haut avec

les mains en rythme, comme s'ils s'apprêtaient à attraper un objet. Contra AHLBERG (1987), p. 64 65, fig. 17. Voir aussi Tölle (1964), p. 43, n 88.

${ }^{13}$ CoOK (1951), p. 46-49, et pl. 10; OHLy (1953), A 20a et A 20, p. 40-44. Aussi Coldstream (2003), p. 123 sq. Pour un parallèle mycénien et même des Cyclades (Naxos), cf. l'hydrie mycénienne de la nécropole de Kamini (Musée de Naxos, 1734) : petite figure dansante aux bras levés dans une file de danseurs. VLACHOPOULOS (1999), p. 89 et n. 95.

${ }^{14}$ Pour de plus grands groupes choraux d'hommes qui "applaudissent " (mais pas au-dessus de la tête), voir WFGNer (1968), n cat. 75, pl. U V, face A. Il est intéressant de constater que, sur l'autre face, apparât un chœur de sept jeunes filles. Cf. aussi WEGNER (1968), n cat. 114, pl. U V et $\mathrm{n}^{\circ}$ cat. 61, pl. U III. Pour un exemple équivalent, voir la zone inférieure du col de l'hydrie proto-attique d'Égine, aujourd'hui à Berlin, où sont représentées les figures masculines aux mains jointes sur le devant, comme s'ils les frappaient. Sur la zone supérieure est représenté un chœur féminin : CVA Berlin 1, pl. 1. Pour des parallèles de danseurs masculins de la région procheorientale, qui joignent leurs mains en "applaudissant" (fin vII" s. av. J.-C.), voir AIGN (1963), p. 175, A/6a; MAAS - MCINTOSH-SNYDER (1989), p. 13, 23, nº́ 14.

15 Cf. aussi le skyphos du Musée archéologique d'Athènes, 784, CVA Athena - Musée arch. Athènes II, 9, pl. 10-11; DAvison (1961), p. 85-86, fig. 134; Crowhurst (1963), p. 49, n 10, 47; TÖLLE (1964), p. 14, 28-29, 36-37 : sur la face intérieure est représenté un chœur mixte; l'une des figures féminines, probablement la chorège, ouvre largement ses bras, comme si elle avait l'intention "d'applaudir ». De même, la demière figure du chour féminin représentée sur la zone supérieure du col de lhydrie proto-attique (voir n. 7) "applaudit » probablement. CVA Berlin 1, pl. 1. Crowhurst (1963), n 13, p. 11 et 281.

${ }^{16}$ XVIII, 494 : hoũ

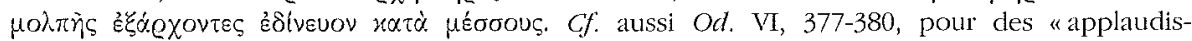
sements" qui accompagnent la danse en rythme. De même, FITTSCHEN (1973); WEST (1992), p. 123.

17 Concernant le sujet de l'appellation des instruments du genre «lyre-cithare », voir WEST (1992), p. 50-51; MATHIESEN (1999), p. 253. 
les genoux fléchis (les chevilles n'arrivent pas aussi haut que celles du danseur du canthare de Copenhague). Il a les mains presque jointes au-dessus de la tête, selon un mouvement analogue à celui des danseurs du pendentif de Paros. Presque le même mouvement, c'est-à-dire un saut vers le haut et des «applaudissements » au-dessus de la tête, est répété par la figure masculine représentée à côté d'une lyre d'une grandeur surnaturelle sur une partie de skyphos géométrique récent provenant d'Érétrie ${ }^{18}$. Si l'on considère les représentations du canthare de Copenhague comme une tentative de figurer les mouvements successifs composant une figure de danse, dont le point culminant serait le mouvement de l'avant-dernière figure" ${ }^{19}$, alors il est possible que les figures sur le pendentif de Paros réunissent, dans une sorte de vision synthétique, des phases successives de la danse qui sont distinguées dans la peinture de vase géométrique récente: les genoux fléchis et la frappe des mains au-dessus de la tête, c'est-à-dire le point de départ (jambes) et le point culminant (bras) d'une seule figure de danse.

Le mouvement des figures du pendentif et des figures comparables de la peinture de vases et de l'orfèvrerie du géométrique récent suit des modèles iconographiques antérieurs provenant du Proche-Orient et de l'Égée préhistorique. Sur l'orthostate gravé des musiciens de Carchemish ${ }^{20}$, daté de la période hittite moyenne (très probablement aux XIV"-XII ${ }^{e}$ siècles), est représentée un petit personnage masculin qui marche sur la pointe des pieds, les genoux légèrement fléchis, et les mains jointes au-dessus de la tête, parmi des musiciens jouant de l'aulos, des percussions et un instrument du type de la pandoura. De même, sur des parties des fresques de Kéa, datées de la période MR IB/HR II et provenant d'un environnement culturellement mixte, mais d'un caractère nettement cycladique, on retrouve des modèles iconographiques de clanseurs comparables: des figures masculines habillées d'un chiton court sont représentées ici aussi la bouche ouverte comme s'ils chantaient. Ils ont les mains levées au-dessus de la tête, les paumes ouvertes, et les genoux, dans le cas où ils sont conservés, fléchis comme s'ils couraient ou sautaient. Peut-être s'agit-il d'une file de danseurs et non d'une danse circulaire. Il est intéressant de noter que le fond pur brun doré est interprété comme une représentation possible d'un espace spécialement aménagé (dans

${ }^{18} N^{\circ}$ inv. 11310. ANdreiomenou (1983), p. 97 et pl. 26 (n 129); AHLBERG (1987), p. 79-80 et fig. 31. Pour l'identification de l'instrument de musique, voir BOARDMAN (1990), p. 367-368.

19 Crowhurst (1963), p. 26 sq., n 32; TÖlle (1964), p. 12-13, n 4, pl. 3; AhlBERG-CornflL (1987), p. 63 sq. et fig. 15. Il est intéressant de constater que la danse est effectuée à proximité d'un autel (c'est ainsi qu'on peut interpréter l'objet situé devant le joueur de lyre) : CrowHurst (1963), p. 28; AHLBERG-Cornkl. (1987), p. 64. Pour des mouvements comparables effectués successivement, cf: l'aryballe corinthien plus récent de Pyrthias, RoEBuck - RoEBUCK (1955), p. $158-163$.

20) WOOLEY (1921), pl. B 17b. Pour la datation, voir WOOLFY (1952), p. 195-197, 202-203, 247 sq. De même, AHLberg (1987), p. 70-71. 
le sens d'une "piste de danse ») et nettoyé de toute trace de végétation ${ }^{21}$. Si nous acceptons que la représentation sur une plaque de marbre provenant du Korphi t'Aroniou de Naxos (cycladique ancien II-III) représente une danse, alors les débuts des mouvements dansants comparables, aux genoux fléchis et aux bras levés, se rencontrent dans la zone des Cyclades dès le III ${ }^{\mathrm{t}}$ millénaire av. J.-C. ${ }^{22}$.

\subsection{Les poissons et l'oiseau}

Le thème de l'oiseau aquatique, soit isolé, soit en combinaison avec le poisson, n'est pas inconnu des représentations de danse de l'art géométrique récent. Sur un fragment de vase de l'Héraion d'Argos apparaît un grand oiseau aquatique avec une svastika sur les ailes (qui sous-entend un mouvement circulaire de danse) à la fin d'un chour de femmes, entre deux personnages masculins. Le première effectue un mouvement de soliste en sautant au-dessus d'une forme triangulaire, alors que le deuxième, dont seules les jambes sont conservées, attend peut-être de faire un mouvement équivalent, à moins qu'il ne s'agisse d'un musicien ${ }^{23}$. De plus, oiseau aquatique et poisson évoquent l'eau, mais aussi le caractère rituel de la représentation sur une partie de cratère géométrique argien. On y trouve un personnage masculin et un cheval debout sur une surface pointillée, très probablement une plage de cailloux, à côté de séries de lignes en zig-zag représentant l'eau de manière imagée. En haut à droite se trouve une procession dansante de quatre hommes portant des palmes. ${ }^{24}$. On peut encore mentionner une onochoé d'Italie (BM 49.5-18.18), une ouvre qui fait écho à des influences eubéennes, représentant une danse mixte que Coldstream identifie comme la geranos, en se fondant, entre autres, sur la présence de cet oiseau aquatique,

${ }^{21}$ Sur les fresques sont également représentées des figures féminines. ABrawowiTz (1980), p. 57 sq. et pl. 4. Voir aussi CASKEY (1971), p. 377-378, spéc. 374-375; CASKEY (1972), p. 357-401. Les fresques ont été trouvées dans un petit édifice près du bastion NE du rempart.

22 Dounas (1966), p. 56-58; Dimakopoulou (1990), p. 116, n $n^{\circ} 112$ : danse ou scène de chasse. Sur la peinture rupestre, les figures dansantes n'«applaudissent» pas. Pour l'utilisation éventuelle de l'établissement du cycladique ancien II à Korphi t'Aroniou en tant que sanctuaire, voir aussi LAMBRINOUdAkIs (1990), p. 99-100. Pour le rapport entre la danse, la chasse et le sacrifice, voir LONSDALE (1995), p. 275, 280 et n. 53, qui se réfère à une fresque néolithique de CatalHüyük représentant une sorte de danse cynégétique.

${ }^{23}$ Crowhurst (1963), no 61, 63, 64, p. 62 sq. et 239 sq.; TÖlle (1964), p. 44 (n 101 ); CoURBIN (1966), p. 159, 437, n 6, 447, pl. 147; WEGNER (1968), n 53, pl. 6d; AHLBERG-CORNELl (1987), p. 65 , fig. 19.

${ }^{24}$ C240, 750-735 av. J.-C. COURBIN (1966), pl. 40; BOARDMAN (1983), fig. 2.4.a, 2.4b. Pour le contenu nartatif de la représentation, voir STANSBURY-O'DONNELL (1985), p. 329-330. LANGDON (1989), p. 198, attribue un contenu religieux à la représentation et la met en rapport avec des pratiques postérieures de sacrifice de chevaux en l'honneur de Poseidon à Argos. Pour les représentations les plus anciennes d'oiseaux et de poissons dans l'art géométrique, voir CARTER (1972), p. 32-33. Plus particulièrement, concernant l'oiseau dans l'art géométrique des Cyclades, cf. CARTER (1972), p. 32-33. Sur les ex-voto d'oiseaux dans des sanctuaires, voir Kourou (1999), p. 69 $s q$. Pour le rapport étroit entre oiseaux et poissons dans l'art mycénien récent, voir Brison (1970), p. 63. 
la grue, qui lui donne son nom ${ }^{25}$. Comme nous allons bientôt le voir, dans le cas du pendentif aussi, l'interprétation de l'image nous permet d'identifier l'oiseau à une grue ${ }^{26}$.

\subsection{Le labyrinthe et les autres décorations linéaires}

Les représentations de labyrinthe circulaire ont souvent été mises en relation avec le symbole très ancien et polysémique de la spirale, mais aussi des méandres, même si, malgré une certaine parenté iconographique, la manière de fabriquer ce genre de labyrinthe suit une logique différente de celles des spirales et des méandres ${ }^{27}$. La base pour la construction de ce dessin est la croix placée au centre et autour de laquelle sont formés sept «corridors » créés par l'union successive de seize points au total, définis par : les branches de la croix, les pointes des quatre angles inscrits dans les quartiers de la croix, et un point au milieu de chaque angle ${ }^{28}$ (sur la figure de la face B sont clairement distingués les liens aux pointes des branches de la

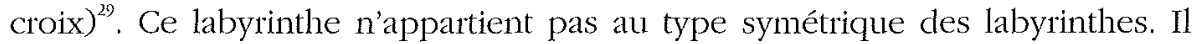
est à sens unique. Parmi les représentations antiques, il y a d'habitude une entrée dans la partie supérieure gauche et le centre se trouve dans la partie inférieure droite de la croix ${ }^{30}$.

La représentation la plus ancienne, me semble-t-il, de ce genre de labyrinthe est datée de la période HR IIIB et présente une forme quadrangulaire, sur

25 COLDSTREAM (1968b), p. 87. Voir aussi BOLANAKi-KONTOLEONTOS (1989), p. 149, n. 16.

26 BENSON (1970), p. 63. Pour des exemples HR de grues, voir pl. XVII, 6.

${ }^{27}$ Pour la symbolique de ce motif, voir COOK (1914), p. 472 sq.; FrontisI-Ducroux (1975), p. 147 sq.; KeRn (1982), p. 41-42. Voir aussi DuCHEMIN (1970), p. 30 sq. Concernant l'extension et l'interprétation des décorations labyrinthiques, voir aussi LEKATSAs (1973). Pour le rapport aux méandres, Heller (1946). Contra RICHARDSON (1966), p. 287 et n. 3 . En tout cas, il faut signaler que le type de spirale le plus proche du labyrinthe circulaire est la double spirale ou spirale composée, qui contient deux tracés en mouvement opposé. Pour les plus anciens exemples de ce type de spirale, voir HeLLfr - CAIRNS (1969), spéc. p. 257 sq. où Heller révise ses précédents points de vue et attribue l'origine du schéma labyrinthique au dessin évolué d'une spirale composé de lignes parallèles.

${ }^{28}$ Il est particulièrement notable que la croix, aux angles et aux points, se rencontre comme une décoration isolée sur des sceaux préhistoriques proche-orientaux, déjà depuis le III" millénaire av. J.-C. : BUCHANAN - MOOREY (1981), n²2, 30-32. Cf. spéc. VON DER OSTEN (1957), $n^{\circ}$ 37. Cf. aussi HOGARTH (1920), pl, V, n 127.

29 Sur les modes de fabrication de ce motif, voir l'analyse de COLTON (1944), fig. 8; HeLler (1946) et (1961), avec toute la bibliographie concenée. Dans l'article de Heller - CAIRNS (1969), p. 243-250, le dessin est expliqué en termes mathématiques. Pour d'autres points de vue concernant l'élaboration du motif, voir aussi RICHARDSON (1966), p. 289, qui le met en relation avec la double hache (dans ce contexte se trouve d'ailleurs, également, le lien du mot labyintbos au mot d'origine orientale labrys). Au contraire, STIEGLITZ (1981), p. 195-198, considère que le mot labyrintbos est un emprunt de l'égyptien lapi-ro-buml qui signifie «Temple of the Mouth of the Sea » et se réfère à l'autel égyptien bien connu sur le lac Moeris.

30 Cf., pour cela, ColToN (1944), p. 129-134. Sur les représentations plus récentes, l'entrée se trouve en-dessous. Sur l'œenochoé de Tragliatella, l'entrée est à gauche. 
la face arrière d'une tablette de Pylos ${ }^{31}$. Une partie de labyrinthe circulaire, analogue à celui du pendentif parien, apparaît aussi sur un fragment de vase provenant de Tell Rifa At en Syrie, mais il n'est pas daté avec certitude ${ }^{32}$. La représentation de ces types de labyrinthe la plus connue, datée avec une relative certitude, se situe sur l'œnochoé étrusque trouvée dans une tombe à Tragliatella, près de Caere, et datée de la deuxième moitié du viI siècle av. J.C. ${ }^{33}$. La représentation de ce labyrinthe, qui porte l'inscription TRUIA sur son cercle extérieur, est liée à l'accomplissement du rite ludus trojae décrit par Virgile et mise en rapport avec le tracé du labyrinthe crétois ${ }^{34}$. Le même type de labyrinthe se rencontre au début du Iv siècle av. J.-C. sur la surface supérieure d'une tuile de l'acropole $e^{35}$, et à partir du troisième quart du IV ${ }^{e} s$. av. J.C., il se répète sur une série de monnaies de Cnossos ${ }^{36}$. Pendant la pétiode hellénistique récente et romaine, nous le retrouvons, dans sa forme rectangulaire, en tant que graffito sur les murs des maisons de Délos ${ }^{37}$ et de Pompéi, où, dans un cas, il s'accompagne d'une inscription explicative LABYRINTHUS (Heller 1961, 61; Kern 1982, 98, n 107, 108). Ce dessin continue à survivre en Europe occidentale pendant le Moyen âge et est probablement transféré en Amérique puisqu'il se rencontre également chez les Indiens Hopi de l'Arizona du Nord (Colton 1944; Heller-Cairns 1969, 238).

Les lignes ondulées sur la face $B$, aussi bien que sur les côtés inférieur et latéraux du pendentif, constituent très probablement des idéogrammes de l'eau. Si cette interprétation est correcte, ces lignes soulignent probablement le rapport du rite représenté sur le pendentif avec l'élément aquatique et accentue l'unité iconographique de toutes les faces de l'objet ${ }^{38}$.

31 LANG (1958), p. 190 (Cn 1287, pl. 46). Comme cela a été signalé par BENNETT - OLIVIER (1973), p. 76-79, la face A est en palimpseste et il n'est donc pas facile de dire si le tracé du labyrinthe peut être mis en relation avec un texte et, dans l'affirmative, lequel. Cf. PALAima (1992), p. 64-65.

32 L'objet n'est pas intégré dans un contexte stratigraphique précis; SETON - WILLIAMS (1961), p. 68-87, pl. $40, n^{\circ} 5$, le classe parmi les trouvailles du niveau III (fin XIII ${ }^{\leftarrow}$ s. av. J.-C.). Cf. aussi KERN (1982), p. 97.

33 SMAll (1986), p. 65. Pour des types de labyrinthes comparables en Italie du nord, datés entre 750-550 av. J.-C., c/. KERN (1982), p. 89-90, n $\mathrm{n}^{\circ} 78-82$.

$3 \dot{~ V I R G i l e, ~ E ̈ n e ́ i d e ~ V, ~ 545-603 . ~ P o u r ~ l ' œ e n o c h o e ́ ~ d e ~ T r a g l i a t e l l a, ~ v o i r ~ H e l l e r ~(1946), ~ p . ~} 128$ sq. Concernant l'inscription, des chercheurs soutiennent que le nom TRUIA ne provient pas de la ville homonyme, mais est lié au verbe latin truare ou amptruare qui signifie «je cours en avant et en arrière dans une danse rituelle, je me meus vivement ». SMaLl (1986), p. 76 sq.

35 KERN (1982), p. 98, $\mathrm{n}^{\circ} 105$, avec bibliographie. Pour son interprétation comme «secret professional mark of craftsman $»$, voir RICHARDSON (1966), p. 287.

${ }^{36}$ Pour l'apparition et l'évolution de ce type de labyrinthe sur les monnaies cnossiennes, voir SMALL (1986), p. 73, et n. 33, avec la bibliographie afférente.

37 De la maison des Tritons: BrLinEau (1978), p. 150, fig. 34; KERN (1982), p. 98, n 105.

36 BOARDMAN (1983), p. 15-36 (avec la bibliographie plus ancienne) où il affirme de manière convaincante que, dans certains cas, les éléments décoratifs ont une signification iconographique définie par le contexte de la représentation. Concernant l'usage du motif des lignes brisées parallèles pour la représentation de l'eau, voir ibid., p. 23, avec des exemples de choeurs. 


\section{L'inter'prétation}

Comme en témoignent le lieu de la découverte et les éléments iconographiques, le pendentif représente l'une des danses les plus connues de l'antiquité, la fameuse geranos. La particularité et l'intérêt exceptionnel du contenu iconographique de ce pendentif résident dans la composition des divers éléments, composition qui se rapproche de ce qu'A. Snodgrass définit comme "récit synoptique», une méthode qui présente plusieurs points communs avec l'expression poétique contemporaine. Par le biais du récit synoptique, une histoire est " concentrée » dans une image qui, sans aucune répétition de personnage, contient et rappelle les étapes multiples de l'action ${ }^{30}$. Dans le cas du pendentif, le récit se développe sur toutes ses faces, et spécialement sur les deux grandes, qui doivent être «lues » comme une unité.

L'argument essentiel contre l'identification de la geranos, c'est-à-dire l'absence d'un chour' mixte, est réfuté, me semble-t-il, par l'interprétation des deux danseurs comme les meneurs de la geranos, lesquels fonctionnent, dans ce cas, comme une représentation raccourcie d'une danse de groupe ${ }^{4 n}$.

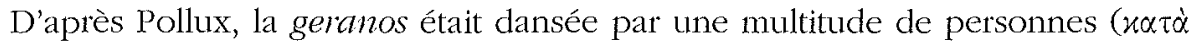
$\pi \lambda \tilde{n} \theta$ os $\dot{\omega} \varrho \chi 0 \tilde{v} \tau o)$ en file, avec deux meneurs aux extrémités ${ }^{41}$, qui devaient jouer un rôle particulier dans le rite ${ }^{42}$. Si nous interprétons ainsi les deux personnages, la décoration centrale ${ }^{43}$ acquiert une signification particulière : d'une part, elle réunit les deux danseurs qui appartiennent au même ensemble choral, étant donné qu'ils ont la même orientation ${ }^{\text {t4 }}$, d'autre part, cependant,

39 SNODGRAss (1987), p. 136 sq.; (1998), p. 57-58, 60-61; STRANSBURY - O'DONNELL (1999), p. 89. Sur la dimension narrative de l'art géométrique, voir récemment STRANSBURY - O'DONNELL (1999), avec une révision des points de vue antérieur's sur le sujet (chap. 1).

40 À propos d'une oenochoé géométrique portant une figure de chœur, COLDSTREAM (1968b), p. 93, signale que cette espèce d'uabréviation » n'est pas du tout rare dans la narration géométrique, du moment où le nombre de figures est défini par la forme du support. Cf. aussi CrowHuRst (1963), p. 4, qui signale que les deux danseurs peuvent représenter un nombre indéterminé de participants. La même chose est valabe pour le chœur des figures portant des vases sur la tête, sur le canthare de Copenhague. Crowhurst (1963), p. 9, n. 5.

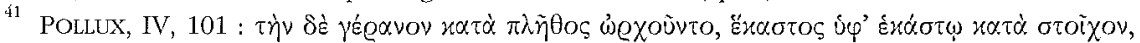

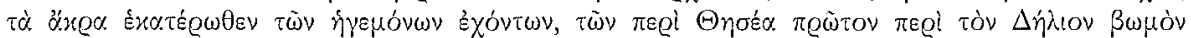

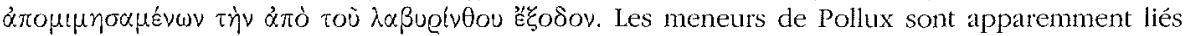
au geranoulkos d'Hésychios. Encore aujourd'hui, les meilleurs danseurs sont placés au début et à la fin d'un groupe de danse.

42 Il n'est pas impossible que le nom de la danse soit lié au rôle joué par les meneurs du groupe, équivalent à celui des chefs dans les essaims d'oiseaux. Pour le rapport au niveau des sons, voir Papadopoulou (1998), p. 203-204.

43. Dans le catalogue du musée de Paros et dans la référence au pendentif par ZaphfiropouLOS (1962), p. 246, elle est interprétée comme un serpent.

4 À propos de l'oenochoé géométrique B4 (2657) de Tübingen (CVA Tübingen 2, pl. 14-15, p. 23-26) où est représenté un grand chour mixte (DAVISON [1961]n o ? 83-84, fig. 127; TöLlE [1964], pl. 1-2; Wegner [1968], Uib; SNODGRass [1998], p. 64 sq., fig. 26), Crowhurst (1963), p. 43, signale que l'artiste marque le grand nombre également par la répétition d'un élément vertical sur le corps du vase. On peut peut-être attribuer une signification analogue à l'usage d'une ligne brisée verticale comme une décoration complémentaire parmi les membres d'un groupe de danse dans certaines représentations géométriques de danses circulaires : cf. par ex. les hydries $n^{\circ} 15$ 
elle les distingue en suggérant qu'ils ne se trouvent pas dans une succession immédiate $^{45}$. Elle fonctionne dès lors, à cause de sa place et de son dessin puisqu'elle suit absolument le mouvement au pied des figures - comme une indication sommaire de l'ensemble des danseurs ${ }^{\text {4́. }}$. Par ailleurs, le genre de mouvement effectué par les personnages plaide aussi pour leur identification comme meneurs, c'est-à-dire le mouvement de soliste avec des sauts et des «applaudissements». Les petites différences qui ont été signalées dans la description des figures renforcent l'idée que la figure de gauche est à identifier avec le dernier danseur du chour, c'est-à-dire le deuxième meneur de la geranos. Ces différences peuvent être inteprétées, soit comme un effort de représentation des figures selon la perspective, soit comme une tentative de représentation d'un personnage plus jeune, ou d'une figure aux membres moins développés (mais pas un enfant qui, selon les conventions iconographiques de l'époque, devrait être représenté à une plus petite échelle), vu que, dans de nombreuses représentations géométriques et plus tardives, le dernier danseur qui effectue des mouvements de soliste paraît être plus jeune $^{47}$. Pour l'interprétation du motif, il est important de noter que la présence d'hommes meneurs (surtout des jeunes) aux extrémités d'un chœur n'exclut pas la participation de membres féminins dans celui-ci ${ }^{48}$. C'est prouvé par des parallèles provenant surtout de l'iconographie argienne du géométrique récent, et proto-attique.

L'identification de la représentation comme geranos est encore renforcée par l'image de l'oiseau aquatique qui donne son nom à la danse. L'oiseau, combiné au poisson, exprime le rapport de la danse avec l'eau, quant au lieu

(Louvre, CA 1333), 16 et 18 (Musée Archéologique d'Athènes, 14423) chez TöLLE (1964), p. 14-16, pl. 5-6. De même Johunsen (1945), fig. 5-6.

${ }^{45}$ À ce sujet, d'intéressantes remarques chez Crowhunst (1963), p. 50, pour les cas où le musicien se distingue du chœur par des décorations supplémentaires.

46 Par ailleurs, nous ne devons pas oublier que la danse anamix ne signifie pas des danseurs placés enallax, mais une participation commune à un rite choral, chose rare pour les données grecques anciennes.

${ }^{47}$ C'est ainsi qu'est interprétée la figure masculine à la fin d'un choeur féminin, très probablement circulaire, sur le tesson provenant de l'Héraion d'Argos (n. 23). Pour des exemples plus tardifs de figures juvéniles à la fin d'un choeur, voir CROwHURST (1963), p. 66-67; DELAVAUX-ROUX (1994), p. 68. La différence dans la représentation des détails anatomiques peut être aussi indicative d'une différence d'âge : $c f$. les figures féminines qui dansent en portant des vases sur la tête sur le canthare NM 727 de Copenhague. AHLBERg (1987), p. 57-59, propose d'identifier la figure de droite - qui n'a pas de poitrine - à une jeune fille. Cependant, cette figure est de taille plus grande.

Voir aussi n. 47. Pour un autre exemple d'un meneur de danse masculin proto-attique, qui "applaudit» en participant au chour féminin, voir l'hydrie 31312, CVA Berlin 1, pl. 40, p. 33; CoOk (1934-1935), p. 176 , pl. 43. Cf aussi à l'époque géométrique récente des vases protoattiques où, sur les deux faces ou sur les deux zones du col, sont représentés, d'une part, un chœur d'hommes «applaudissant», d'autre part, un chœur féminin circulaire: WEGNER (1968), $\mathrm{n}^{\circ}$ cat. 75, pl. U V; CVA Berlin 1, pl. 1; Crowhurst (1963), p. 32 sq. Voir supra, notes 7 et 14. 
de l'exécution (mer, lac, fleuve), quant à la qualité des danseurs (voyageurs), quant au caractère de la danse (rapport à la mer et protection des dangers) ${ }^{49}$.

D’une importance égale pour l'identification est la représentation du labyrinthe, le plus ancien labyrinthe circulaire dans le bassin de l'Égée. Dans le contexte choral représenté sur le pendentif, le labyrinthe indique manifestement deux choses :

1) Un lieu d'accomplissement du rite, une " piste de danse " équivalant à celle que Dédale ${ }^{50}$ aurait aménagée (Il. XVIII, 590) ${ }^{51}$. Des pistes circulaires de ce genre ont probablement existé en Crète pendant le MR III, comme en témoigne la découverte d'une grande plateforme circulaire et de deux petites à Cnossos par l'École archéologique anglaise ${ }^{52}$. En plus, des représentations de labyrinthes d'origine minoenne liées à un espace spécialement aménagé pour la représentation d'un spectacle, comme par exemple des rites acrobatiques, se rencontrent dans l'espace géographique de la Méditerranée orientale à partir du MM II et III en Crète ${ }^{53}$. Par ailleurs, dans les exemples des fresques à caractère égéen de Tell el-Dab'a d'Égypte, datées au début de la XVIII dynastie, le fond labyrinthique est lié à des spectacles rituels athlétiques et acrobatiques $^{54}$.

2) Un parcours choral précis. Il est possible que ce schéma de labyrinthe, mis à part le lieu de l'exécution du rite, imprime visuellement, dans une sorte de

(9) Pour d'autres interprétations de l'oiseau (par ex. comme symbole solaire), voir CourBiN (1966), p. 478 sq., avec les points de vue antérieurs. De même, BOARDMAn (1983), p. 19.

50 Dans le sens local et surtout en rapport avec le "chœur" "que Dédale avait construit pour Ariane, a été interprété le mot da-da-re-jo-de (Daidaleion) sur une tablette en Linéaire $\mathrm{B}$ de Cnossos. KERÉnYi (1968), p. 1023-1025. Voir aussi BURNS (1974-1975), p. 1-12; MorRIS (1995), p. 73 sq., 186 et n. 138.

51 Pour ce passage, voir CaLAME (1977), p. 52 (avec des étymologies et des parétymologies du mot depuis l'antiquité), et plus récemment LonsDale (1995), p. 273, et surtout n. 3. Le fait que le mot choros indique aussi le lieu de la danse est attesté depuis l'antiquité (Od. VIII, 260; PAUSANIAS, III, 11, 9; scbolies à l'lliade XVIII 590 a, b Erbse IV). L'indication d'un parcours tracé par des lignes sur le sol n'est pas inconnue, au moins pour l'antiquité tardive : sur l'orchestra d'époque romaine du théâtre de Dionysos à Athènes, il y a une mosaïque en méandre qui facilitait probablement le mouvement du chœur à cet endroit. L'explication d'Hésychlos $s . u$.

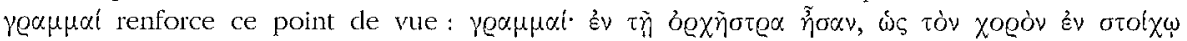

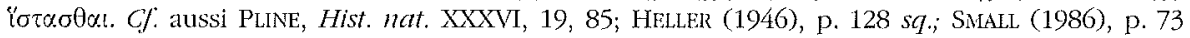
$s q$. p. 14 .

52 WARREN (1984), p. 307-323, pl. 30-35. Ces édifices datent du MR III. Cf. Morris (1995),

53 Phaistos, MM II : IMMErwaHr (1990), p. 22-23, fig. 6d. Knossos, MM III : Evans (1921), p. 357, fig. 256. Evans met en rapport les représentations labyrinthiques de Crète avec les figures labyrinthiques égyptiennes (fig. 258a-c) datées de la VI dynastie et suivantes. RICHARDSON (1966), p. 285-286, distingue correctement les décorations labyrinthiques minoennes et égyptiennes avec leur éventuel symbolisme (anaktoron, édifice souterrain, etc.) du type de labyrinthe à la croix sans exclure, bien sûr, que de tels motifs aient contribué à sa formation (mais pas à sa naissance).

54 Je dois cette remarque à A. VLACHOPOUlos. Voir aussi BIETAK - NARTNATOS - PALYVOU (2000). MORGAN (1995), p. 29-53, et SCHAw (1995), p. 106-113, interpiètent la combinaison entre l'acrobate au taureau et le motif du labyrinthe en relation avec le palais cnossien. Voir aussi MARINATOS (1998), p. 84 sq., fig. 24. 
vue d'en haut, le mouvement des danseurs" ${ }^{55}$. Le labyrinthe est formé par le mouvement de deux "files » contraires et polymorphes qu'impriment dans l'espace et le temps les files des danseurs, en désignant «le chemin le plus long enfermé dans l'espace le plus court $»^{56}$. Les deux parcours surgissent, soit parce que, à un moment précis de la danse, le groupe se divise en deux parties qui s'entremêlent ${ }^{57}$, s'entrecroisent, soit parce que le mouvement de la danse est renversé. Le dernier devient alors le premier, comme le sous-entend la référence de Pollux à deux meneurs ${ }^{58}$.

La théorie selon laquelle la décoration labyrinthique imprime des mouvements chorégraphiques nous aide à interpréter le tracé hélicoïdal au-dessus du labyrinthe circulaire. Ce schéma représente, dans une forme plus longue, le début du parcours de l'une des lignes (semi-chour, vers la droite) du labyrinthe circulaire, une sorte de prélude au rite de danse ${ }^{59}$. En considérant attentivement ce parcours, nous constatons qu'il ne s'agit pas d'un cercle, mais d'un mouvement boustrophédon qui rend visuellement les verbes $\varepsilon \lambda i \sigma \sigma \omega, \pi \alpha \varrho \alpha \lambda \lambda \dot{\alpha} \sigma \sigma \omega, \dot{\alpha} v \varepsilon \lambda i \sigma \sigma \omega$ utilisés par les sources anciennes pour indiquer l'imitation du mouvement du labyrinthe par les interprètes de la geranos ${ }^{(0)}$.

En « lisant» sous cet angle interprétatif les éléments iconographiques composant le pendentif, nous constatons que l'artisan réussit à «raconter» le chœur et à représenter ses changements avec succès, en se servant de la

55 Roux (1979), p. 118, comprend le motif en tant que chorégraphie; voir aussi VoLANAKIKontoleontos (1989), p. 147 et n. 12. Pour des parallèles ethnographiques de danses de type labyrinthique et leur interprétation, voir SACHs (1938), p. 88.

56 C'est la danse elle-même qui est en file et qui renvoie au fil d'Ariane: Frontist-Ducroux (1975), p. $145 s q . ;$ Detienne (1983), p. 541 sq.

${ }^{57}$ C'est fondé sur cette idée d'ailleurs que des représentations chorales comme celles des vases proto-attiques ou la danse sur le lébès gamikos à figures rouges du peintre de Syriskos (Musée de Mykonos, 970, provenant de Rhénée) ont été mises en rapport avec la geranos par certains chercheurs : COLDSTREAM (1968b), p. 92; Délos XXI, 31-32, p1. 5-7, 57; Crowhurst (1963), p. 295, 300-301. Pour le tissage des fils, voir scholies à l'lliade XVIII, 590 Dindorf II : yogòv

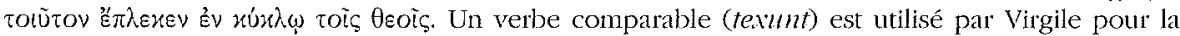
description du ludus troiae (Ën. V, 580 sq.).

58 FronTISI-DUCROUX (1975), p. 150; ROUX (1979), p. 118-119; DETIENNE (1983), p. 547-552.

59 Lá manière de composer les deux figures, un tracé labyrinthique en forme de goutte et un labyrinthe circulaire, renvoie au schéma que SCHWEITzER (1971), p. 26, appelle "géométrisation du motif du poulpe», peut-être le plus important de la période proto-géométrique. Pour des parallèles iconographiques de ce motif provenant de Paros, voir BERrANGER (1992), pl. 20 (cratère de Koukounaries HR IIIC). Si effectivement il existe un lien iconographique, alors de la dégradation de cette décoration mycénienne pendant la période proto-géométrique, nous passons à un nouvel usage, enrichi d'un contenu complètement différent.

(6) Cf. aussi le mot $x \alpha \mu \pi \eta \dot{~ c h e z ~ A p O L L O D O R E ~(I I I, ~ 1, ~ 4), ~ p o u r ~ l e ~ l a b y r i n t h e ~ e n ~ C r e ̀ t e ~(x \alpha \mu \pi \alpha i \varsigma ~}$

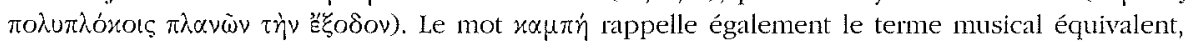
la $\chi \alpha \mu \pi \dot{\eta}$ dans le sens de la métatropie. Une image comparable au mouvement boustrophédon est créée par l'orientation opposée des danseurs d'une frise à l'atutre sur l'«amphore du chœur" en relief, conservée de manière fragmentaire, provenant de Xombourgo de Tinos, datée autour de 700 av. J.-C. et mise en rapport avec la représentation d'une danse labyrinthique, probablement la geranos. Musée de Tinos B 63. KONTOLEON (1969), p. 227, pl. 48-50; VOlANAKI-KONTELEONTOS (1989), p. 149. 
division des surfaces du pendentif comme d'un moyen d'exprimer la succession temporelle et l'évolution pendant le rite ${ }^{6}$. Sur la face A est représenté, de manière concise ${ }^{62}$, le temps présent, le bic et nunc, un "instantané » de l'acte de danse : les deux meneurs accomplissent une danse mise en rapport avec l'élément marin. Le fait que les deux figures sont disposées l'une après l'autre dans la même orientation montre que la composition est ouverte. Cela signifie qu'elle nous suggère la continuation et l'évolution du mouvement ${ }^{63}$ : en se portant sur la face $B$ clu pendentif, on a la possibilité de voir la manière dont le chœur se développe, à savoir ses phases suivantes ou précédentes, ce qu'on appelle une prolepse ou une analepse narrative dans une représentation concise $^{64}$.

La variété de l'orientation des figures et des décorations contribue à la narration de manière aussi efficace: d'une part, le mouvement de deux figures guide le spectateur vers la droite (vers la face B), mais en même temps la différenciation dans l'orientation du poisson et surtout le mouvement de tête de l'oiseau aquatique - conventionnel pour l'iconographie géométrique contient l'élément du renversement du sens de la danse, du mouvement double qui se développera complètement dans les schémas labyrinthiques de la face $\mathrm{B}^{65}$. Il est encore possible que des éléments comme la double extrémité des lignes ondulées ${ }^{66}$ constituent une sorte de «prolepse narrative » et signifient donc le mouvement double, qui change potentiellement de schéma et d'orientation'. Le point culminant de la narration est le labyrinthe circulaire - un thème iconographique mycénien qui survit dans l'espace cycladique et qui s'adapte au contexte rituel et mythologique du culte délien ${ }^{68}$. Le labyrinthe synthétise, non seulement le parcours du chœur, qui avait manifestement un

61 SNODGRass (1998), p. 55 sq. et 64 sq. (plus particulièrement pour l'attribution de mouvements successifs au chcur'). D'après STANSBURY-O'DONnEll (1999), p. 231, des représentations qui, d'une certaine manière, «fragmentent " l'action parce qu'elle ne peut pas être entièrement visible si l'on ne tourne pas l'objet sur lequel elle figure (comme c'est par exemple le cas avec les aryballes corinthiens), introduisent le sens de la succession temporelle, autant dans la vision que dans la narration.

62 Pour la signification de la concision dans les représentations géométriques, voir WEBSTER (1955), p. 48.

6.3 Il fonctionne dès lors aussi comme un «indice temporel " (time index): STANSBURYO'DONNFLL (1999), p. 29.

${ }^{64}$ C'est ainsi que STANSBURY-O'DONNELL (1999), p. 89, analyse l'anachronisme narratif.

is Pour l'évocation du mouvement dans une représentation narative, voir STANSBURYO'DONNELL (1999), p. 79. Étant donné qu'aucun élément du pendentif ne paraît posséder un simple caractère décoratif, même les fentes hémisphériques sur sa partie supérieure peuvent avoir une fonction équivalente à celle des dessins gravés.

${ }^{66}$ Une double extrémité existe aussi d'après le dessin de la publication, à l'une des extrémités du labyrinthe circulaire provenant de Tell Rifa At (SETON-WILLIIAMs [1961], pl. XL, 5). Ce détail n'est pas distingué chez KERN (1982), p. 97, $\mathrm{n}^{\circ} 102$, où le dessin du tesson est moins fidèle que celui de la publication originale.

${ }^{67}$ Pour la sémiologie de la narration dans la peinture de vases grecque ancienne, voir récemment STANSBURY-O'DONnELl (1999), p. 13 sq. et 43 sq. (pour l'anachronisme narratif).

68 Cf. des remarques équivalentes de Morris (1995), p. 188 concernant la spirale. 
caractère apotropaïque, mais aussi la triple signification du mot choros: " piste de danse» (lieu), «mouvement et évolution du chœur» (temps), "groupe de danseurs" (sujet) ${ }^{69}$. Il est notable que le matériau du pendentif, qui, quand il se met en contact avec le corps humain, acquiert une transparence particulière, permette une lecture «synchronique » de ses côtés, au-delà de la lecture successive. En tournant le pendentif vers la lumière, on peut « lire » ses différents côtés en même temps, étant donné que l'un se projette sur l'autre. Les danseurs paraissent danser dans le labyrinthe, les poissons nager dans l'eau.

La manière de raconter la geranos sur le pendentif renvoie à son parallèle iconographique le plus important, c'est-à-dire la scène du choeur d'Ariane du bouclier d'Achille ${ }^{70}$, qui est présentée en Iliade XVIII, 590-606 $6^{71}$, liée à la geranos depuis l'antiquité. Je ne veux pas dire par là que le pendentif, à une époque aussi ancienne, représente l'une des scènes du poème homérique ${ }^{72}$. Je crois pourtant qu'il y a un lien, tant dans certains éléments chorographiques des deux scènes, qui se rapportent manifestement à une tradition commune de danse, que dans leur mode de narration, iconographique et poétique ${ }^{73}$.

La représentation du chœur décrite par le poème homérique est assez compliquée, comportant plus d'une étape; logiquement, on s'attendrait à ce qu'elle soit une danse labyrinthique du type de la geranos. Elle comporte des éléments qui se développent de manière concise sur les deux faces du pendentif de Paros: d'une part, un mouvement de groupe en cercle qui, à un moment donné, se divise, se retourne, tourne à l'envers, pour redevenir un cercle par après, d'autre part, les mouvements de soliste des meneurs ${ }^{7 /}$

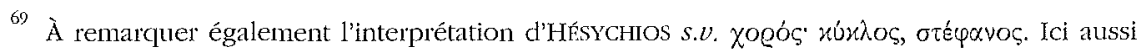
le rapport avec la Crète, Ariane (stepbanos) et le labyrinthe est évident. Cf. Morris (1995), p. 188.

70 Comme il a été signalé, l'intérêt particulier de la description homérique réside, d'une part, dans le fait qu'il s'agit de la plus ancienne description d'une ouvre d'art iconographique et, d'autre part, dans le fait qu'il reflète un mode de "lecture» de telles ouvres par les spectateurs de leur époque. Sur cette problématique, voir récemment STANSBURY-O'DONNELL (1999), et plus particulièrement par rapport au bouclier, p. $42 \mathrm{sq}$.

${ }^{71}$ Concemant l'authenticité de ces vers, voir la bibliographie du sujet chez STANSBURYO'DONNELL (1999), p. 315, n, 2 et 3.

72 Cf. FITTSCHEN (1973). Pour la représentation des scènes homériques, et plus généralement, épiques, sur les vases géométriques, voir AHLBERG-CORNELL (1992). A. SNODGrass (1998) se montre plus sceptique sur le sujet de la représentation des scènes homériques pendant le viH" $\mathrm{s}$. av. J.-C. Voir aussi STANsBury-O'Donnell (1999), p. 31-44; MAZARAKIS-Ainian (2000), chap. 7. Pour l'identification de sujets homériques sur des parties de scènes iconographiques déjà pendant l'âge du Bronze, voir MORRIS (1989), p. 511-535.

73 Dès l'antiquité ont été signalés des parallèles entre la poésie (spécialement épique et tragique) et l'iconographie, alors que la notion de mimèsis (représentation), dans les deux genres, a attiré l'attention de Platon et d'Aristote. D'après Simonide (chez PluTARque, Mor, 346e):

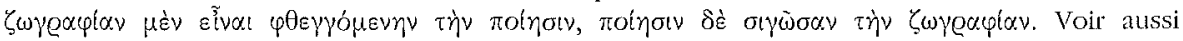
STANSBURY-O'DONNELL (1995), p. 319; (1999), p. 11-114.

${ }^{74}$ Le fait que la geranos comportait des éléments de chorégraphie de solistes est indirectement suggéré par des sources plus tardives. C'est seulement ainsi que l'on peut comprendre pourquoi Lucien inclut la geranos dans la catégorie des clanses vulgaires aux sauts très hauts, etc.

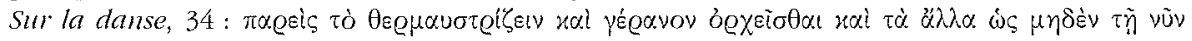


(kybistètêrôn), qui mènent le chant ${ }^{75}$. Ce chour, comme l'ensemble du bouclier d'Achille, est entouré par Okéanos ${ }^{76}$. De la même manière, toutes les faces du pendentif sont entourées par des idéogrammes aquatiques qui, accompagnés des poissons et de l'oiseau, soulignent le caractère marin de la danse $^{77}$. Les équivalences entre la dernière clanse du bouclier et la geranos labyrinthique s'étendent aussi à la symbolique de leurs mouvements chorégraphiques dont le modèle, dès l'antiquité, a été recherché dans le mouvement des étoiles $^{78}$. La danse des vers 590-606 du chant XVIII de l'Iliade, avec les mouvements doubles, antithétiques, et successifs du cercle et de la file, du mouvement de droite à gauche, et inversement, a été considérée, premièrement, comme imprimant la conception homérique du temps (lequel n'est pas seulement linéaire, mais cyclique, comme il arrive dans les représentations géométriques ${ }^{79}$, et, deuxièmement, concentrant l'essence du bouclier d'Achille, interprété comme imago mundi, comme l'harmonie des contraires ${ }^{80}$. Les mouvements opposés du chœur, dans toutes ses étapes ${ }^{81}$, acquièrent dès lors des dimensions cosmiques, ils se rapportent aux mouvements successifs du ciel diurne et nocturne, et se trouvent en accord avec le principe du bouclier au centre duquel est placé le cosmos (la terre, la mer et le ciel avec le soleil, la lune et les astres en mouvement circulaire) ${ }^{82}$. Des interprétations équivalentes ont été attribuées tant aux chours labyrinthiques ${ }^{83}$, qu'aux déco-

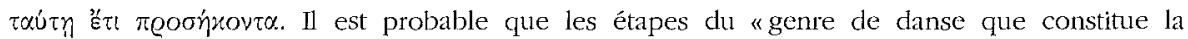

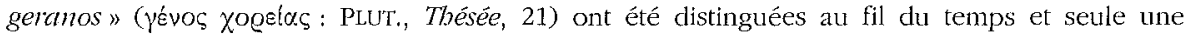
partie de la danse était encore considérée comme geranos du temps de Lucien.

$75 C f$. l'attribution éventuelle d'une chanson aux danseurs du pendentif.

${ }^{76}$ D'ailleurs, pour le rapport entre la geranos et les tites liés à la mer est indicatif d'Eustathe qui, en commentant le chœur du bouclier et en le rapportant à celui que Dédale a enseigné à Cnossos, remarque que plusieurs marins, même de son temps, accomplissent une danse aux circonvolutions multiples et complexes, qui imitent les mouvements compliqués du labyrinthe.

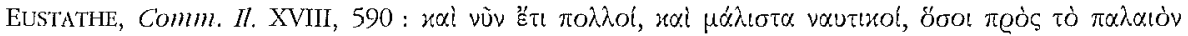

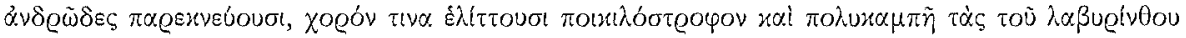

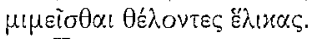

77 Pour le rapport de la grue avec les voyages marins, voir ARISTOTE, Hist. Anim., 597a 30-32. La grue, oiseau de la sagesse, se distingue par sa capacité de traverser le ciel, comme un marin qui sait réunir dans son voyage les confins du monde, le nord au sud, traverser un lieu sans point de repère, sans voie, comme la mer. Detienne (1983), p. 548-549; PAPAdopoulou (1998), p. 179 $s q$.

78 AuBRTOT (1999), p. 42 sq.

79 Pour le temps non linéaire de la narration poétique et iconographique, voir STANSBURYO'DONNELL (1995), p. 330.

81 TAPLIN (1980), p. 1-21, et plus récemment AuBriot (1999), p. 53-56, avec la bibliographie complète. Voil aussi NAGY (1997).

${ }^{81}$ Même dans le cas du mouvement des meneurs, qui reflète les cercles des danseurs en

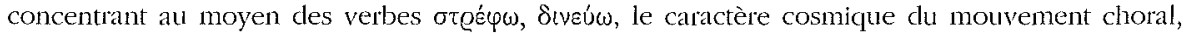
Aubriot (1999), p. 50 et n. 163.

82 Aubriot (1999), p. 45 sq. Il est intéressant que la Grande Ourse, autour de laquelle tournent les autres corps célestes, alors que celle-ci reste immobile, s'appelle Hélikè. Voir aussi EDWARDS (1989), p. 280-281; AUBRIOT (1999), p. 52, n. 169.

83 MArus ViCTORINus, A $\%$. Gramm. (Keil VI, p. 60), lie la danse de Thésée aux mouvements de l'univers et comprend musicalement, dans le sens de la strophe et de l'antistrophe, les 
rations labyrinthiques et spiroïdales mises en rapport avec la symbolique solaire et astrale (comme par exemple avec la double spirale interprétée comme le mouvement céleste du jour et de la nuit, ou le cycle de la vie et de la mort, etc. $)^{81}$. Bien que de telles interprétations puissent susciter plusieurs oppositions, il faut souligner qu'elles s'harmonisent avec le contenu de la geranos en tant que rite initiatique courotrophe lié aux éléments de la mort et de la renaissance, à travers la réactualisation mimétique des dangers inhérents à tout passage symbolique et réel, que ce soit un voyage maritime, que ce soit un passage vers l'âge adulte ${ }^{85}$.

\section{La datation, le lieu de fabrication et l'usage}

Comme nous l'avons dit plus haut, le pendentif a été trouvé pendant des travaux d'aménagement du Délion. Il n'existe donc aucune donnée de fouille qui pourrait aider à la datation.

Sa forme ne trouve pas de parallèles. Elle présente quelques ressemblances avec des sceaux et des pendentifs provenant d'Égine, de Rhodes et d'Éphèse ${ }^{86}$, qui sont pourtant, soit sans décoration, soit avec une décoration simple, le plus souvent (sur les grandes surfaces ou sur la base). Elles présentent un seul trou de suspension et une forme plus simple, puisque sur la partie supérieure, il n'existe pas de fente hémisphérique ${ }^{87}$. Les formes de ces sceaux datés autour de 700 av. J.-C. tirent leur origine du Proche-Orient. Le pendentif de Paros pourrait être daté de la même période. Son contenu iconographique et, plus spécialement, la manière de représenter les deux

tournants complexes du labyrinthe. De même, les scholies au v. 647 de l'Hécube d'Euripide mettent en rapport la strophe et l'antistrophe dans le chour tragique avec le mouvement des corps célestes. Pour le fragment, voir aussi LAWLER (1967), p. 12-16, qui le rapproche du dithyrambe. Pour la danse des corps célestes, voir aussi LUCIEN, Sur la danse, 7. Pour la geranos,

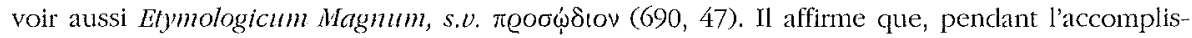
sement de l'hyporchèma, un genre de danse mimétique ayant fleuri particulièrement à Délos, et dans lequel s'inscrivent des danses du genre de la geranos, les danseurs se meuvent de gauche à droite, et inversement, en imitant le mouvement du cycle zodiaque et clu ciel, alors que, à la fin, ils contournent tout l'autel.

${ }^{8-1}$ Signalons aussi que le mathématicien d'Apeiranthos de Naxos M. Bardanis a interprété les doubles spirales paléo-cycladiques représentées sur des peintures rupestres de Naxos en rapport avec le mouvement du soleil : la spirale primordiale montre son mouvement pendant le jour (lumière, vie), la seconde, la nuit (obscurité, enfers). De même, dans d'autres motifs, Bardanis a reconnu des symboles de constellations. BARDANIS (1989), p. 433-446; DOUMAs (1990), p. 158. Pour des interprétations du motif du labyrinthe en tant qu'idéogramme du mouvement du soleil et son rapport avec le changement des saisons, voir KERN (1982), p. 41-42.

85 CALAME (1977), p. 225-231; VOLANAKI-KONTOLEONTOS (1989); DELAVAUX-ROUX (1994), p. 80; PAPADOPOUlou (1998), p. $152 \mathrm{sq}$. Pour la bibliographie antérieure relative à la geranos, voir NAEREBOUT (1997), p. 131-132.

86 FURTWÄNGLER (1906), pl. 118, 2-6; JACOPI (1932-1933), p. 327, fig. 72-VI; BOARDMAN (1963), p. $141, \mathrm{n}^{\circ} \mathrm{M} 57, \mathrm{M} 58$; HOGARTH (1908), p. 190, pl. 37, 4-5.

87 Des fentes plus simples dans leur partie supérieure apparaissent pourtant sur certains sceaux hittites datés du II millénaire av. J.-C. HogarTh (1920), fig. 15, 16, nº cat. 128, 129, 142 , 146,211, mais toujours avec un trou. 
danseurs de la face A s'accordent aussi avec cette datation. Les personnages et l'oiseau sont figurés d'une manière qui imite la technique de l'ombrage alors que les poissons sont représentés en fonction de leur contour. Qui plus est, l'artisan réussit à copier plus fidèlement lá manière de représenter les figures dans la céramique du géométrique récent, en choisissant des manières différentes de graver la pierre. Pour les membres supérieurs, et surtout les membres inférieurs, des figures humaines, la gravure est profonde et d'une coupe triangulaire (la main droite de la figure de gauche est sortie de son tracé pendant la gravure et, par conséquent, elle apparaît plus large). Par contre, le torse et la tête des figures, ainsi que le corps de l'oiseau, sont représentés en surface.

Il est bien connu que l'attribution des sceaux à un atelier précis n'est pas facile, étant donné que, comme le signale J. Boardman, les caractéristiques techniques des ateliers sont habituellement assez individuelles et d'une durée de vie limitée ${ }^{88}$. Sur le pendentif de Paros, nous pouvons repérer des éléments provenant des traditions de l'Égée et du Proche-Orient, ainsi que d'ateliers continentaux du géométrique récent. Pourtant, le caractère cycladique reste très marqué puisque sa fabrication suit des directives rituelles liées au culte d'Apollon Délios ${ }^{89}$. Il présente dès lors des traits caractéristiques qui s'accordent avec la danse qu'il évoque, laquelle unit par la chorographie le cercle et la ligne droite, le masculin et le féminin, et par le mythe, les diverses routes de l'Égée ${ }^{90}$, tout en restant cependant entiètement intégré dans le cadre du culte délien.

La découverte de la plus ancienne représentation de la geranos à ce jour, dans le Délion de Paros ${ }^{91}$, apporte de nouvelles données pour identifier le lieu

88 BOARDMAN (1963), p. 110-111; COLDSTREAM (2003), p. 151.

89 Il faudrait toutefois signaler que, autant la tradition mythique que la pratique cultuelle lient la geranos, non seulement à Apollon, mais aussi à Artémis. Selon le mythe, Thésée, lors de son départ pour la Crète, avait invoqué - d'après Phérécyde - Apollon Oulios et Artémis Oulia, que le héros avait remerciés pour son salut en rentrant à Délos (MACk, Sal. I, 17, 21; FGrHist 3 F 149. GaLlFt DE SANTERIRE [1958], p. 186; Farnell [1907], p. 234 et n. 238). Le rapport étroit des deux divinités avec la danse est confímé par son lieu d'exécution (le keratinos bômos), qui reflète la parenté des deux divinités, tant du point de vue topographique (puisqu'il se trouve entre leurs deux temples à Délos) que du point de vue mythique (l'autel a été fabriqué par Apollon avec les cornes des chèvres tuées par Artémis). Pour des éléments supplémentaires attestant le lien de la geranos avec Aitémis, voir PAPADOPOULOU (1998), p. 223 sq.

") VOLANAKI-KONTOLEONTOS (1989), p. 153.

91 La divinité adorée avec certitude, au moins à partir du $v^{*} s$. et après, est, d'après les témoignages épignaphiques, Artémis Délia, mais il existe des éléments qui attestent la présence d'un sanctuaire d'Apollon dans le même lieu (comme des bornes d'un sanctuaire d'Apollon Délios trouvées non loin du Délion et provenant probablement de cet endroit [/G XII 5 , 214; ZAPHEIROPOULOS (1962), p. 245; RUBENSOHN (1962); BERRANGFR (1992), p. 81 sq.; ZAPHEIROPOULOU (1998), p. 22]). A ces éléments s'ajoute le pendentif que nous examinons ici. Comme d'autres l'ont déjà signalé, l'un des traits caractéristiques du culte délien des deux divinités est le rapport étroit entre elles, exprimé tant dans le mythe et dans les rites (tout particulièrement les choeurs) que dans la topographie de leur sanctuaire. BURKFrT (1985), p. 144; VOLANAKI-KONTOLEONTOS (1989), p. 147 (pour la geranos); PApadopoulou (1998), p. 48 sq. (pour le mythe des Hyperbo- 
d'apparition et d'efflorescence de cette danse, qui semble se situer dans les Cyclades. Par ailleurs, elle constitue un indice supplémentaire du fait que certains rites, et plus spécialement les choroi en l'honneur d'Apollon Délios en tant que "schémas choraux ", "pistes de danse ${ }^{93}$ et "groupes choraux ${ }^{94}$ - n'étaient pas liés exclusivement à Délos, mais apparaissaient également dans les sanctuaires " déliens »" en dehors de Délos.

Par le caractère à la fois marin et apotropaïque de la danse qu'il représente, le pendentif est classé parmi les sceaux utilisés en tant qu'«amulettes ${ }^{96}$, comme c'était le cas de deux exemples géométriques de la même catégorie trouvés par $O$. Rubensohn dans les fouilles du Délion ${ }^{07}$. Comme en témoignent les traces d'usure, le pendentif doit avoir été porté pendant très longtemps. Nous ne pouvons pas savoir s'il était propriété du temple et faisait partie de l'équipement des responsables du rite, ou s'il a été dédié à la divinité comme offrande d'action de grâce, à la manière de la danse qu'il représente. Il est cependant certain que la force poétique de la narration iconographique et son symbolisme complexe le classent parmi les rares exemples de l'art géométrique cycladique, une création digne du dieu auquel il était destiné.

Zozie PAPADOPOULOU

1, Neofronos

GR - 16121 ATHÈNES

réens), 226 (pour la geranos), 348-352 (pour les Déliades). Pour la topographie des sanctuaires des deux divinités à Délos, voir Bruneau - Ducat (1983), p. 118-160.

92 Pour une exécution probable de la geranos à Athènes, en Crète et dans les Cyclades (Naxos), voir Delavaux-Roux (1994), p. 77. Notons qu'une danse au nom de ageranos est attestée jusqu'à nos jours dans les Cyclades et plus particulièrement à Paros.

93 LONSDALE (1995), p. 280, considère que le transport du xoanon d'Aphrodite de Grète à Délos et son emplacement à côté du keratinos bômos d'origine crétoise indiquent que le lieu sacré du rite choral peut être transféré.

)f Papadopoulou (1998), p. 310 sq. Aussi Papadopoulou-Belmehd - PAPADOPOUlou (2002), pour les Déliades en dehors de Délos. Le choeur réactualise la présence divine et assure la continuité de la faveur divine à l'endroit où il est exécuté. Voir surtout LONSDALE (1993), p. 119121.

95 Pour le caractère supra-local d'Apollon Délios, voir LAMBRINOUDAKIS (2001), p. 19 et n. 57.

\% Cf. aussi WALDSTEIN (1905), p. 345, qui, à propos des amulettes de l'Héraion d'Argos, se demande si ce genre d'objet était fabriqué par des attisans ou par les prêtres afin d'être distribués aux fidèles.

${ }^{97}$ Le premier porte une décoration linéaire et provient probablement d'un atelier cycladique, alors que le deuxième, portant une image de cheval, est probablement importé : RuBENSOHN (1962), p. 80; BOARDMNN (1963), p. 143 , n० 6 et 7. 

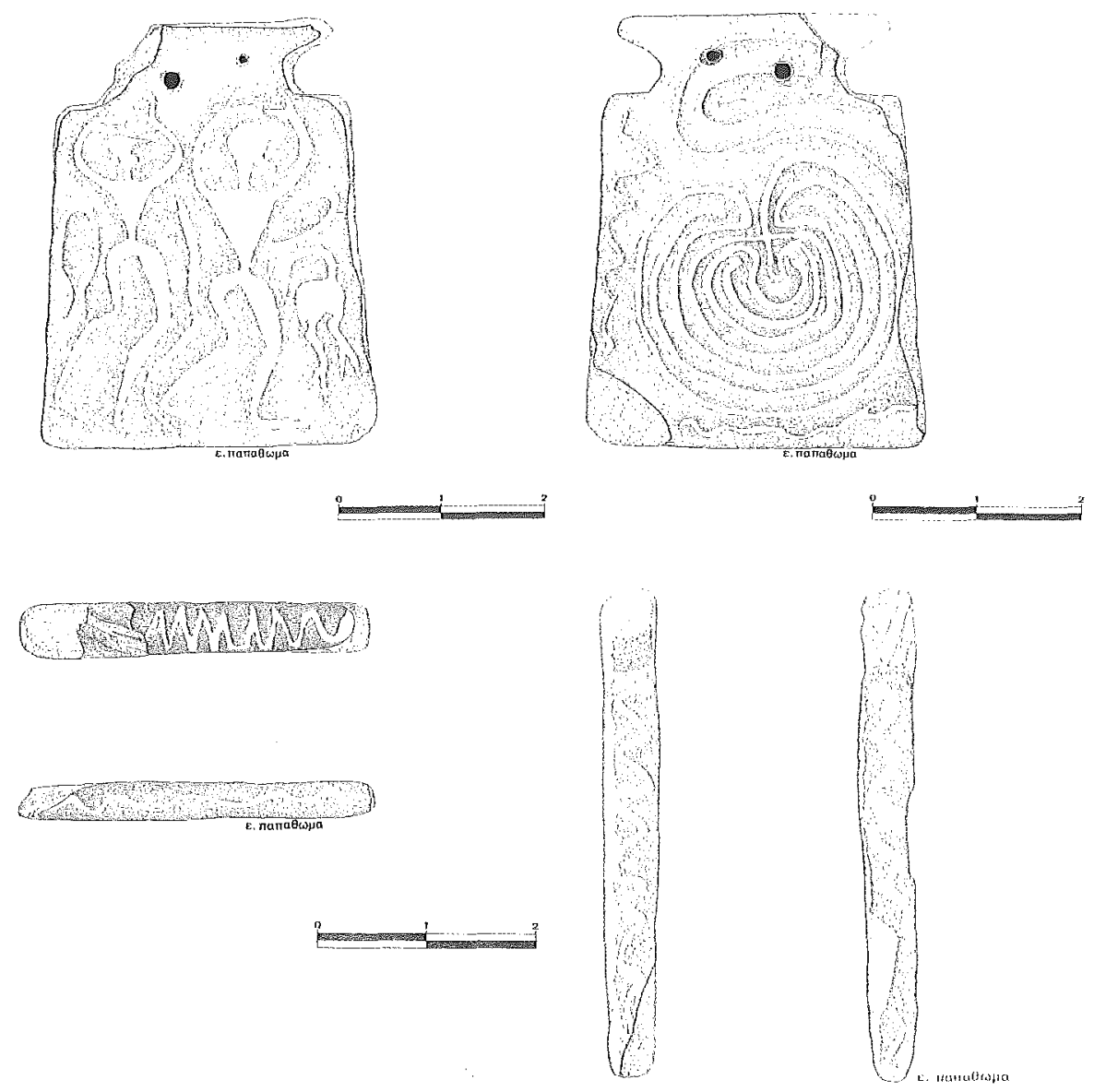

\section{Bibliographie}

Abrhmowitz (1980) : K. ABRamowtrz, «Frescos from Ayia Irini, Keos. Parts II-IV », Hesperia 49 , p. $57-85$.

AHLBerg (1971) : G. AHLBERg, Prothesis and Ekphora in Greek Geometric Art, Göteborg (SIMA 32). AHLBERG-CORNELL (1987): G. AHLBERG-CORNELl, «Games, Play and Performance in Greek Geometric Art. The Kantharos Copenhagen NM 727 Reconsidered », Acta Archaeologica 58, p. 55-86.

AHLBERG-CORNELl (1992): G. AHLBERG-CORNELL, Myth and Epos in Eally Greek Alt: Representalion and Interpretation, Jonsered (SIMA 100).

AJGN (1963) : B. AJGN, Die Gescbichte der Musikinstrumenle des Agäiscben Ratumes bis um 700 vor Christus, Frankfurt am Main.

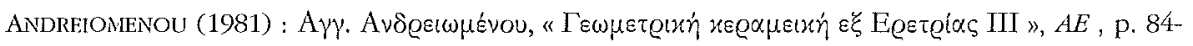
113. 
Aubrtot (1999) : D. Aubriot, "Imago Iliadis. Le Bouclier d'Achille et la poésie de l'Tliade ", Kenos 12, p. 9-56.

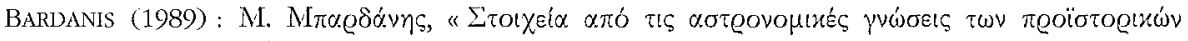

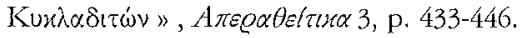

BennetT - OLIVER (1973) : E.L. BenNeiT, Jr, J.-P. Ouvier, The Pylos Tablets Transcribed 1, Roma (Incunabula Giaeca 51).

BENSON (1970) : J. BENSON, Horse, Bird and Man, Amherst.

Berranger (1992): D. Berranger, Recherches sur l'bistoire el la prosopographie de paros à l'époque archaïque, Clermont-Ferrand.

BEVAN (1989) : E. BEVAN, « Water-birds and the Olympian Gods », ABSA 84, p. 163-169.

BOARDMAN (1963) : J. BOARDMAN, Island Gems. A sludy of Greek Seals in the Geomalric and Early Arcbaic Periods, London.

BOARDMAN (1970): J. BOARDMAN, Greek Gems and Finger Rings. Early Bronze Age to Late Classical, London.

BOARDMAN (1983) : J. BOARDMaN, «Symbol and story in Geometric art ", in W.G. MoON (éd.), Ancient Greek Ant and Iconography, Wisconsin, p. 15-36.

BOARDMAN (1990) : J. BOARDMAN, "Chariot, Trapeze or Lyre? ", Oxford Joumal of Archaeolog" 9, p. $367-368$.

Bruneau (1978) : Ph. Bruneau, «Déliaca II.23. Dessins à lîlot de la Maison des comédiens », $B C H 102$, p. 147-151.

Bruneau - Ducat (1983) : Ph. Bruneau, J. Ducat, Guide de Délos, Paris.

BUCHAN - MOOREy (1981) : B. BUCHANAN, P.R.S. MOOREy (éds), Calalogue of Ancient Nen Eastem Seals in thw Ashmolean Mliseum, II, The Prebistoric Stamp Seals, Oxford.

Burkert (1985) : W. BuRKerT, Greek Religion (transl. J. Raffan), Cambridge MA, Harvard University Press.

Burns (1974-1975) : A. Burns, «A chorus of Ariadne », CJ 70, p. 1-12.

CALAME (1977): Cl. CALANE, Les chceurs de jeumes filles en Grèce archaïque I, Rome.

CARTER (1972) : J. CARTER, "Narrative art in the geometric period », ABSA 67, p. 25-58.

CARTER - MORris (1995) : J.B. CarTer, S.P. Morris (éds), The Ages of Homer. A Tribute to Emily Tounsend Vermeule, Austin.

CASKEY (1971) : J.L. CASKEY, «Investigations in Keos, Part I: Excavations and explorations, 19661970 ». Hesperia 40, p. 359-396.

CASKEY (1972) : J.L. CASKEY, «Investigations in Keos, Part II: A conspectus of the pottery », Hesperia 41, p. 357-401.

Coldstream (1968a) : J.N. Coldstram, Greek Geometric Pottery: A Survey of Ten Local Styles and theit Chronology, London.

COLDSTREAin (1968b) : J.N. COLdSTREAM, «A Figured Geometric Oinochoe from Italy », BICS 15, p. $86-96$.

COLDStreAm (2003): J.N. Colstream. Geometric Greece. 900-700 B.C., London / New York, Routledge.

ColTon (1944) : H.S. Colton, "Troy town on the Hopi Mesas », Scientific Montbly 58, p. 129-134.

CoOk (1914) : A.B. CoOK, Zeus, A Study in Ancient Religion I, Cambridge.

COOK (1934-1935) : J.M. CoOK, « Protoattic Pottery », ABSA 35, p. 165-219.

COOK (1951) : J. COOK, «A geometric amphora and gold band», ABSA 46, p. 45-52.

COUnBin (1966) : P. CourBin, La céramique géométrique de l'Argolide, Paris (BEFAR 208).

CROWHURST (1963) : R. CROWHURST, Representations of Performance of Choral Lyic on the Gleek Monuments, 800-330 B.C., London.

DAVSON (1961) : J.M. DAVISON, Attic Geometric Workshops (Yale Classical Studies 16).

DFlavauX-Roux (1994): M.-H. DElAvauX-Roux, Les danses pacifiques en Grèce antique, Aix-enProvince.

DETIENNE (1983) : M. DetIEnNe, « La grue et le labyrinthe », MEFRA 95, p. 541-553.

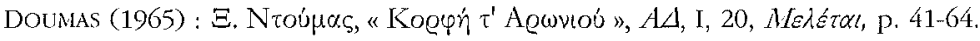


DuchEmin (1970) : J. DuchEmin, "Le thème du héros au labyrinthe dans la vie de Thésée ", $K \omega \varkappa \alpha \lambda \circ \varsigma 16$, p. $30-52$.

EDWarDs (1989) : M.W. EDWARDS, Homer; Poel of the Iliad, Baltimore / London.

Evans (1921) : A.J. Evans, The Palace of Minos at Knossos 1, London.

FARNELL (1907) : L.R. FARNELL. The Cults of the Greek States IV, Oxford.

FitTSCHEN (1973) : K. FitTSCHEn, Der Schild des Acbilleas. Archaeologia Homerica, Kapitel N, Göttingen.

Frontisi-Ducroux (1975): F. Frontisi-Ducroux, Dédale. Mytbologie de l'artisan en Grèce ancienne, Paris.

Furtwängler (1906) : A. Furtwängler, Aegina. Das Heiligtum der Aphaia, München.

Gallet de SaNterre (1958) : H. Gallet de SANTERre, Délos primitive el archä̈que, Paris.

Heller (1946) : J.L. HeLLER, «Labyrinth or Troy Town », CJ 42, p. 123-139.

Heller (1961) : J.L. Heiler, « A labyrinth from Pylos?, AJA 65, p. 57-62.

Heller - CAirns (1969) : J.L. Heller, S.S. CAirNs, "To draw a labyrinth", in Classical Studies Presented to Ben Edwin Perry, Illinois Studies in Language and Literature 58, p. 236-262.

Hogarth (1908) : D.G. Hogarth, Excavations al Ephesus. The archaic Artemisia, London.

HOGARTH (1920): D.G. HOGARTH, Hittite Seals with particular reference to the Asbmolean Collection, Oxford.

Imerwahr (1990) : S. Imminahr, Aegean Painting in the Bronze Age, Pennsylvania / London.

JACOPI (1932-1933) : J. JACOPI, Esplorazione archaeologica di Camiro II (Clara Rhodos V-VII).

Johansen (1945) : F. Johansen, Tbésée et la danse à Délos, Kopenhavn.

KERÉNYT (1968) : K. KERÉnYT, "Möglicher Sinn von di-wo-nu-so-jo und da-da-re-jo-de ", Atti $e$ memorie del $1^{\circ}$ Congresso internazionale di micenologia, 1967, II, Roma, p. 1021-1026.

KRRN (1982), H. KERN, Labyrintbe. Erscbeinungsformen und Deulungen 5000 Jabre Gegenwart eines Urbilds, München.

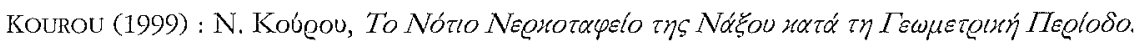

LAMBRINOUDAKIS (1983): V. LAMBRINOUDAKIS, "Les ateliers de céramique géometrique et orientalisante de Naxos : perspectives pour l'analyse archéométrique ", dans Les Cjclades. Malériaux pour une étude de géographie bistorique. Table ronde réunie à l'Université de Dijon les 11, 12 et 13 mars 1982 (CNRS 1983), p. 166-175.

LAMBRINOUDAKIS (2001) : V. LAMBRINOUDAKIS, "The emergence of the city-state of Naxos in the Aegean, in M.C. LENTINI (éd.) The two Naxos cities: a fine link between the Alegean sea and Sicily, Palermo, p. 13-22.

LANG (1958) : M. LANG, «The Palace of Nestor's Excavations of 1957. Part II », A/A 62, p. 181-191.

LANGDON (1989) : S. IANGDON, "The Return of the Horse Leader" " $A / A$ 93, p. 185-201.

LAWLER (1967): L. LAWLR , "Cosmic dance and dithyramb", Studies in bonour of Ullman, presented to bim on the occasion of his $75 \mathrm{lh}$ birthday, Saint-Louis (The Classical Bulletin), p. $12-16$.

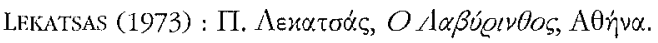

LONSDALE (1993) : S. LONSDALE, Dance and Ritual Play in Greek Religion, Baltimore.

LONSDALE (1995): S.H. LONSDALE, «A dancing Floor for Ariadne (lliad 18.590-592): Aspects of Ritual Movement in Homer and Minoan Religion », in CARTER - MORris (1995), p. 273-284.

MAAS - MCINTOSH-SNTDER (1989) : M. MAAS, J. MCINTOSH-SNYDER, Stringed Instruments of Ancient Greece, New Haven / London.

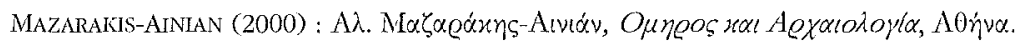

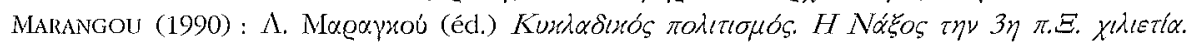
A $\theta \dot{n} v \alpha$.

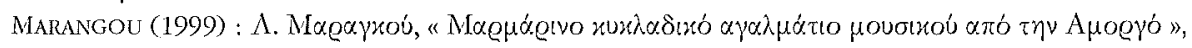
in STAMPOLIDES (1999) p. 20-29.

Marinatos (1998) : N. Marinatos, "The Tell el-Dabca paintings: A Study to Pictorial Tradition", Agypten and Levante 8, p. 83-99. 
Mathesen (1999) : Th.J. MAthiesen, Apollo's Lyre. Greek Music and Music Theory in Anliquily and the Middle Ages, University of Nebraska Press.

MENiCHETTI (1992) : M. MENICHETTI, "L'oinochoe di Tragliatella: mito e rito tra Grecia ed Etruria ", Ostralea 1, p. 7-30.

Morgan (1995) : L. Morgan, «Minoan Painting and Egypt. The Case of Tell el-Dab ${ }^{\mathrm{c}}$ » , in W.V. Davirs, L. Schoficud (éds), Egypt, the Aegean and the Levant. Interconnections in the Second Millenium B.C., London, p. 29-53,

MORris (1989): S.P. MORris, "The miniature frescoes from Thera and the origins of greek poetry », $A / A$ 93, p. 511-535.

MORRIS (1995) : S.P. MORRIs, Daidalos and the Origins of Greek Art, Princeton

NAGY (1990) : G. NAGY, Pindar's Homer. The Ijric Possession of an Epic Past. Baltimore, London.

NAGY (1997): G. NAGY, "The Shield of Achilles: Ends of the lliads and Beginnings of the Polis » in S. LANGDON (éd.) New Ligh on a Dark Age: Exploring the Cullure of Geometric Greece, Columbia, p. 194-207.

Nabrebout (1997): F.G. NaErebout, Athractive Performances, Ancient Greek Dance: Three pretiminay Studies, Amsterdam.

OHLY (1953) : D. OHLY, Griechische Goldbleche des 8. Jabrbunderts v. Cbr., Berlin.

Oтто (1977) : B. Orto, "The Ornamental Motifs of the Cycladic Neolithic and Early Bronze Ages », in THLMe (1977), p. 137-141.

Palaima (1992): Th. G. Palaima, "Mycenaean Scribal Aesthetics ", in EIKSN. Aegean Bronze Age Iconography: Shaping a Methodology. Proceedings of the $4^{\text {th }}$ International Aegean Conference. University of Tasmania, Hobait, Australia, 6-9 April 1992, Liège (Aegaeum 8), p. 6374.

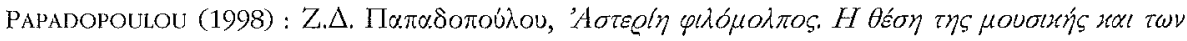

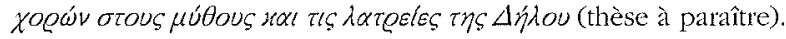

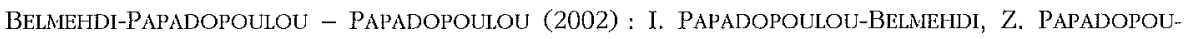
LOU, "Musique et culte : le cas de Délos », in Fr. LABRQUE (éd.), Religions méditerranéennes et orientales de l'Antiquité, Le Caire.

Richardoson (1966): L.J.D. Richardson, "The Labyrinth", L.R. PALAmR, J. Chadwick (éds), Proceedings of the Cambridge Colloquitum on wycenaean Studies, p. 285-299.

ROBERT (1939) : F. Robert, Thymélé, Paris.

RoEBUCK - Roebuck (1955) : M. \& C. RoebuCK, «A Prize Aryballos », Hesperia 24, p. 158-163.

Roux (1979) : G. Roux, "Le vrai temple d'Apollon à Délos ", BCH 103, p. 109-135.

Rubensohn (1962) : O. RubEnsohn, Das Delion von Paros, Wiesbaden.

SaCHS (1938): C. SACHS, Histoire de la danse (trad. L. Kerr), Paris.

SCHWEITZER (1971) : B. SCHwEITZER, Greek Geomelric Am (trad. C. Usborne), London.

Seton-Wiluams (1961) : M.V. SeTon-Williams, "Preliminary Report on the excavations at Tell Rifa At $\gg, \operatorname{Iraq} 23$, p. 68-87.

SHAW (1995) : M.C. SHAw, "Bull Leaping Frescoes at Knossos and their Influence an the Tell elDabCa Murals ", Agupten und Levante 5, p. 91-120.

SMALl (1986) : J.P. SMALL, «The Tragliatella Oinochoe », Röm. Mitt. 93, p. 63-96.

Snodgruss (1987) : A. SNoDgrass. An Archaeology of Greece: the Present Stale and the Future Scope of a Discipline, Berkley / Los Angeles / London.

SNODGRass (1998): A. SNOdGRASs, Homer and the Aitists. Text and Picture in Early Greek Art, Cambridge University Press.

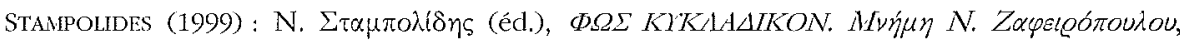
A $\theta$ ñvo.

STANSBURY-O'DONNELl (1995) : M.D. S'TANSBURY-O'DONNELL, « Reading Pictorial Narrative: The Law Court Scene of the Shield of Achilles », in CARTER - MORkIS (1995), p. 315-334.

Stansbury-O'Donnell (1999) : M.D. StAnsbury-O'DOnNell, Piclorial Narative in Ancient Greek Ant, Cambridge University Press. 
STIEglitz (1981) : R.R. STIEgLITZ, « Labyrinth: Anatolian Axe ot Egyptian Edifice? », in L. CASSON, M. Pricf: (éds), Coins, Cullure and History in the Ancient World; Numismatic and Otber Studies in Honor of B.L.Trell, Detroit, p. 195-198.

TAPLIN (1980) : O. TAPLIN, "The Shield of Achilles within the Iliad », $G \& R 27$, p. 1-21.

Thimme (1977) : J. Thimme (éd.), Art and Culture of the Cyclades, Handbook of an ancient civilisation (trad. P. Getz-Preziosi), Karlsruhe.

TÖLLE (1964) : R. TöLLE, Frügriechische Reigentänze, Waldsassen / Bayern.

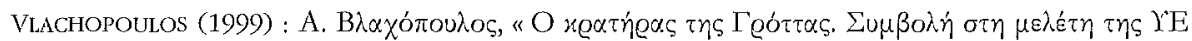

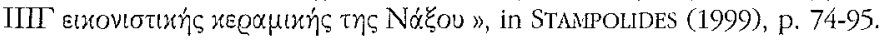

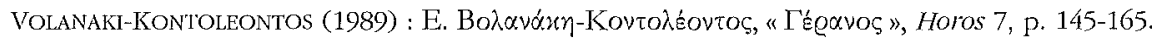

VON DER OSTEN (1957) : H.H. VON DER OSTEN, Altorientalische Siegelsteine Sammlung Hans Silvius von Aulock, Uppsala.

WALDSTEIN (1905) : Ch. WhLDSTEIN, Argive Heraeum II, Boston / New York.

WARREN (1984) : P. WARREN, "Circular platforms at Minoan Knossos », ABSA 79, p. $307-323$.

Webster (1955) : T.B.L. WeBster, "Homer and Attic Geometric Vases », ABSA 50, p. 38-50.

WEGNER (1949) : M. WEGNER, Das Musikleben der Griechen, Berlin.

Wegner (1968) : M. WFGNER, Musik und Tanz. Archaeologia Homerica II U, Göttingen.

WEST (1992) : M.L. WEST, Ancient Greek Music, Oxford.

WOOLEY (1921) : C.L. WOOLLEY, Carchemish, Report on the Excavations at Jerablus on behalf of the British Musetum, II: The Toun Defenses, London.

WOOLEY (1952) : L. WOOLEY, Carbemish, The Excavation in the Inner Town, III, London.

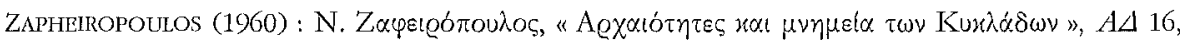
Elovixá, p. 244-251.

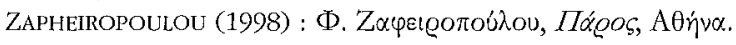

